\title{
VORWORT DES HERAUSGEBERS ZUM 2. BAND
}

Der lange Zeitraum, der zwischen dem Abschluß der beiden Bände des RL liegt, dürfte den Wunsch, auch dem 2. Band ein Geleitwort mit auf den Weg zu geben, genügend gerechtfertigt erscheinen lassen. Dagegen bedarf die lange Dauer der Drucklegung des 2. Bandes selbst einer Rechtfertigung. Die Gründe liegen zunächst in den Arbeitsbedingungen des Herausgebers, die zeitweilig noch ungünstiger waren als beim 1. Band. Vor allem aber war das starke Mißverhältnis zwischen diesen Bedingungen und der zu leistenden Arbeit an der Verzögerung schuld. Es mußte mit dem Fortschreiten des Druckes notwendigerweise immer größer werden. Das Bestreben, das Manuskript auf der Höhe der Forschung zu halten, beanspruchte um so mehr Arbeit, je länger der Druck sich hinzog, die Drucklegung aber verlangsamte sich um so mehr, je mehr Arbeit das Manuskript erforderte. Freilich wäre Schrader selbst sicher schneller vorangekommen. Das liegt im Wesen der Sache. Schrader hätte sich überdies höchstwahrscheinlich im wesentlichen mit der technischen Korrektur begnügt, wie es bei den Bogen der Fall war, die wir noch zusammen herausgebracht haben. Der Verfasser ist eben dem Herausgeber gegenüber auch in dieser Hinsicht im Vorteil. Er braucht nur die Verantwortung vor sich und der Wissenschaft zu übernehmen, der Herausgeber muß dazu noch die Verantwortung vor dem Autor tragen.

Dieser Umstand stellte den Herausgeber sehr bald vor zwei zeitraubende und höchst unerquickliche Aufgaben, mit denen von vornherein nicht gerechnet worden war, die sich aber als eine unerläßliche Vorarbeit und Vorbedingung der weiteren Herausgabe des Manuskripts nicht umgehen ließen. Mehrfache Stichproben ergaben nämlich die Notwendigkeit, sämtliche Quellen- und Literaturzitate auf ihre bibliographische und sachliche Richtigkeit nachzuprüfen. Was das bedeutet, braucht dem Wissenden nicht gesagt zu werden. Es ist mehr als einmal vorgekommen, daß die Richtigstellung eines einzigen ungenauen oder irrigen Zitats, besonders, wenn es nicht aus einer primären Quelle geschöpft war, einen ganzen Nachmittag angestrengten Suchens auf der Bibliothek erforderte. Erst in der letzten Zeit hat mir Herr stud. phil. Steinmet $z$ diese die Zeit für wichtigere Dinge stehlende Arbeit mit liebenswürdiger 
Hilfsbereitschaft abgenommen, für die ich ihm auch an dieser Stelle danken möchte. Die zweite Aufgabe fiel mir bis zuletzt allein zu: Es mußte auch das gesamte Wortmaterial in verschiedner Hinsicht einer Nachprüfung unterzogen werden. Die Durchführung stieß allerdings auf erhebliche Schwierigkeiten. Die Hilfsmittel der Breslauer Bibliothek reichten hier vielfach nicht aus, und eine Benutzung fremder Bibliotheken und des Austauschverkehrs konnte aus äußeren Gründen gar nicht oder nur in sehr beschränktem Umfang in Frage kommen.

Erst neben und nach diesen beiden der elementaren Richtigkeit und Zuverlässigkeit des Manuskripts dienenden Arbeiten konnte die eigentliche und wichtigste Aufgabe in Angriff genommen werden, durch Um- und Neugestaltung des Textes das Manuskript auf der Höhe zu halten. Auch hier gingen die Anforderungen schließlich über das Maß des Erfüllbaren hinaus. Allein schon die bei dem großen Stoffgebiet des RL so außerordentlich weitverzweigte Literatur zu verfolgen, wuchs sich bei der raschen und ständigen Steigerung der literarischen Produktion in den Nachkriegsjahren zu einer Aufgabe aus, die notwendigerweise eine Einschränkung erfahren mußte, wenn nicht die beiden anderen eben erwähnten Aufgaben, die ja von viel unmittelbarerer Bedeutung und Notwendigkeit waren, darüber zu kurz kommen 'sollten.

Daher bin ich auch gern der Anregung des Verlages gefolgt, mich seit dem Erscheinen des Ebertschen Reallexikons der Vorgeschichte nach der archäologisch-prähistorischen Seite hin auf das Notwendigste zu beschränken. Daß nun der Ebert und der Schrader nebeneinander vorliegen, ist sehr zu begrüßen. Die beiden Werke weisen auf der einen Seite mancherlei Berührungen auf, andererseits weichen sie ihren Zielen und ihrem Inhalt nàch doch wieder völlig voneinander $a b$. Gerade dadurch aber ergänzen sie sich aufs beste. So glaubte ich, diesen Glücksumstand im Sinne einer gesunden Arbeitsteilung und im Interesse des rascheren Fortschritts in der Drucklegung des Schrader ausnützen zu dürfen. Eine gewisse Erleichterung bedeutete das doch.

Aber mochten alle diese Vorarbeiten noch so zeitraubend und zum großen Teil auch unerquicklich sein, die eigentliche Schwierigkeit begann doch' erst bei der Frage, wieweit und in welcher Weise neue Ergebnisse und abweichende Auffassungen Verwertung finden sollten. Der Herausgeber steht ja in seinem Verhältnis zu dem Werk, das ihm anvertraut ist, in der Mitte zwischen dem Benutzer und dem Verfasser. Auf der einen Seite muß er sich die objektiven Forderungen und Bedürfnisse des Benutzers zum Maßstab nehmen, auf der anderen Seite muß er dem subjektiven Recht des Autors Geltung verschaffen. Und gerade Otto Schrader, der als Herausgeber des Hehnschen Werkes den Text des Autors mit so viel pietätvoller Schonung bewahrt hat, konnte die gleiche Pietät für sich selbst beanspruchen und hat es auch ausdrücklich getan. So habe ich mich, wie ich es schon im Vorwort zum I. Band betont habe, nach Möglichkeit auf Żusätze und Angaben neuerer Literatur beschränkt. Die Schattenseiten dieses. Verfahrens waren mir von Anfang an bewußt, aber ich sehe auch heute noch keinen anderen Weg, um die Interessen des Benutzers und des Verfassers in gleicher Weise zu wahren. Nur dort habe ich geglaubt, 
dem ausdrücklichen Wunsch des Verfassers entgegen zu größeren Um- und Neugestaltungen des Textes schreiten zu müssen, wo die Spannung zwischen der Auffassung des Autors und dem Stand der Forschung bezw. zu meiner eigenen Auffassung zu stark erschien oder wo, es das augenblickliche Interesse an bestimmten Problemen geboten erscheinem ließ. Immer aber mußte dabei ein weiterer Gesichtspunkt berücksichtigt werden, der überhaupt ein sehr wesentliches Motiv zu vorsichtiger Zurückhaltung gegenüber Textänderungen bedeutete. Bei den vielfältigen Verknüpfung:en der einzelnen Artikel untereinander und den zahlreichen Verweisungen von einem Artikel auf den anderen bestand die mit dem Fortschreiten des Druckes immer mehr sich steigernde Gefahr, daß Eingriffe in den Text einzelner Artikel unausgleichbare Widersprüche mit bereits gedruckten Artikeln ergebren könnten. Diese Gefahr hätte natürlich vermieden werden können, wenn es möglich gewesen wäre, vor der Fortsetzung des Druckes zunächst noch einmal das ${ }^{\circ}$ Manuskript als Ganzes durchzuarbeiten. Das war aber aus verlagstechnischen Gründen nicht durchführbar. Schließlich kam zu diesen sachlichen und gefühlsmäßigen Momenten noch die Erwägung hinzu, welche praktischen Folgen eine weitergehende Umund Neugestaltung des Textes nach sich gezogen hätte. Für den Herausgeber wäre es ja viel lockender gewesen, statt der ermüdenden und reizlosen Arbeit des Nachprüfens der Zitate und des. Wortmaterials sich in die Probleme zu vertiefen, die in reicher Fülle rechts und links vom Wege auftauchten. Diesen Lockungen nachzugeben, war psychologisch die größte Gefahr. Denn dadurch wäre zumal bei den Abgründen, die-sich so leicht bei Problemen auftun, der Abschluß des Werkes auf weitere unabsehbare Zeit hinausgezögert und damit praktısch überhaupt in Frage gestellt worden. Denn ein solches Vorgehen hätte nichts anderes bedeutet, als das RL in beträchtlichem Umfange neu zu schreiben. Das wäre aber auch gegen die Absichten des Autors gewesen, und ganz abgesehen davon wird man es dem Herausgeber nicht verargen, wenn er zum Abschluß kommen wollte.

Denn volle Befriedigung und Dank bringt die Tätigkeit des Herausgebers gewiß nicht. Dafür muß und kann ihn aber das Bewußstsein entschädigen, zu seinem schwachen Teil das Erscheinen eines Werkes ermöglicht zu haben, das die Früchte eines besonders reiçhen Gelehrtenlebens in sich birgt. Und daß es sich gelohnt hat, 1o Jahre der Arbeit im wesentlichen diesem Werk zu widmen, das hat mir das Interesse bewiesen, das man dem Fortschreiten der Herausgabe in reichem Maße entgegengebracht hat. Bei den vielen inneren und äußeren Hemmungen und Schwierigkeiten, die die Arbeit begleiteten, war dieses Interesse ebenso wertvoll und wohltuend wie die tatkräftige Hilfe und Förderung, die ich von so vielen Seiten erfahren habe. Gerade wenn der Weg zurückgelegt ist, denkt man mit besonderer Dankbarkeit der Weggenossen, die helfend, mahnend oder auch warnend bis zum Ziel mitgegangen sind, und man denkt mit besonderer Wehmut derer, die unterwegs zur ewigen Rast eingekehrt sind. $\mathrm{Zu}$ ihnen gehört auch $\mathrm{Fr}$. Kluge, der Treueste der Treuen. Allen diesen Helfern möchte ich noch einmal, und sei es nur in stillem Gedenken, meinen herzlichen und aufrichtigen Dank aussprechen. $\mathrm{Er}$ 
gilt in erster Linie den schon im Vorwort zum I. Band Genannten. Ich benutze dabei die Gelegenheit, einen Irrtum richtig zu stellen, der dort unterlaufen ist: Es muß statt Dr. v. d. Hagen Dr. B. v. Hagen heißen. Weiterhin muß ich Geh.-R. Hillebrandt $\dagger$, den Proff. Herbig $\dagger$, Malten, Seger, Grünenthal und Frau Prof. Nelly Hilpert danken, ebenso Herrn stud. phil. Witte, der die mühevolle Arbeit des Wortregisters bereitwillig übernommen hat.

Dieses Wortregister war schon ein Wunsch Schraders. Daß er schließlich doch noch verwirklicht werden konnte, ist im wesentlichen dem Entgegenkommen des Verlages zu verdanken, der überhaupt eine nie erlahmende Sorgfalt und Opferfreudigkeit bewiesen hat, für die ich auch an dieser Stelle meinen aufrichtigen Dank aussprechen möchte. Endlich ist es mir ein Bedürfnis, den Beamten der Breslauer Universitätsbibliothek für die jederzeit mit gleicher Hilfsbereitṣchaft und gleichem Verständnis gewährte Unterstützung öffentlich zu danken.

Damit wäre auch die letzte Pflicht des Herausgebers erfültt. Aber ich möchte doch die Feder nicht aus der Hand legen, ohne zum Schluß noch einmal des Mannes zu gedenken, dessen Werk die Arbeit gegolten hat. Jetzt, da sie abgeschlossen ist, drängt sich besonders lebhaft die Frage auf, ob der Autor dieses Lieblingskind seiner Forscherarbeit noch als das seine anerkennen möchte. Würde es ihm zu wenig Fleisch von seinem Fleisch und Blut von seinem Blut dünken oder würde er es im Gegenteil zu wenig entwickelt finden? Aber Otto Schrader ist Zeit seines Lebens ein "gütiger und nachsichtiger Kritiker gewesen, und vor allem ein Mensch, der in seiner großen Bescheidenheit stets seine Person hinter der Sache zurücktreten ließ. So hoffe $i c h$, daß er über die Mängel gütig hinwegsehen und daß er das Fremde, das trotz des Bemühens, dem Werk die persönliche Note und Eigenart zu erhalten, in das RL hineingekommen ist, ohne persönliches Bedauern hinnehmen würde, weil es aus dem Bestreben erwachsen ist, der Sache zu dienen. So möge sein Hauptwerk nun endlich vollendet hinausgehen und möge sein Andenken lebendig erhalten. Wir aber, die wir die Früchte seiner Arbeit genießen, werden ihm in seinem Sinne am besten danken, wenn wir aus seinem Lebenswerk vor allem Anregung und Ansporn zu weiterem Forschen gewinnen, auf daß spätere Generationen sagen können, Otto Schraders Werk war nicht nur Frucht, sondern auch Samen.

$$
\text { Breslau, im Juli } 1928 .
$$

A. Nehring. 